\title{
Finite-Element Modeling of Liquid-Crystal Hydrodynamics With a Variable Degree of Order
}

\author{
Richard James, Eero Willman, F. Aníbal Fernández, Member, IEEE, and Sally E. Day, Member, IEEE
}

\begin{abstract}
A finite-element model of liquid-crystal hydrodynamics based on the Qian and Sheng formulation has been developed. This formulation is a generalization of the Ericksen-Leslie theory to include variations in the order parameter, allowing for a proper description of disclinations. The present implementation is well suited to treat properly the various length scales necessary to model large regions yet resolve the rapid variations in the order parameter in proximity to disclinations.
\end{abstract}

Index Terms-Finite elements, liquid-crystal (LC) modeling, variable order parameter.

\section{INTRODUCTION}

I N COMMON liquid-crystal (LC) structures, relaxation dynamics can be greatly affected by the flow of LC. In fact, the (back) flow immediately after a holding voltage is removed can cause molecules to tilt further and accelerate the subsequent relaxation to a homogeneous state [1]. Ericksen-Leslie theory [2], [3] has been applied to the study of backflow with much success. In this theory, the orientation of LC is represented by a director field, and, thus, the equations are restricted to a uniaxial state with a constant degree of order.

However, when high holding voltages are applied, the order parameter drops, and the biaxial ordering increases near the alignment layers [4]. Similarly, these effects occur in the vicinity of disclinations. The Qian and Sheng formulation [4] is a generalization of the Ericksen-Leslie theory that takes such changes into account. Instead of representing the molecular orientation by directors, the $Q$ tensor, which is a rank-two symmetric tensor, is used. The Beris-Edwards equations [5] are a similar generalization but consider only two viscosity coefficients. Furthermore, the viscosity coefficients in the Qian and Sheng formulation can be obtained directly from the Ericksen-Leslie coefficients, which is the standard means to characterize LCs.

The effect of hydrodynamics on the movement of disclination lines has been investigated by Tóth et al. [6] and Svenšek and Z̆umer [7]. Tóth et al. use a lattice Boltzmann Algorithm to approximate the Beris-Edwards equations. Svenšek and Žumer use a finite-difference discretization of the Qian and Sheng equations. Similar discretizations of these governing equations can be found in [8] and [9].

Manuscript received October 28, 2005; revised April 3, 2006. This work was supported by the Engineering and Physical Sciences Research Council (EPSRC), U.K. The review of this paper was arranged by Editor H.-S. Tae.

The authors are with the Department of Electronic and Electrical Engineering, University College London, Torrington Place, London WC1E 7JE, U.K. (e-mail: r.james@ee.ucl.ac.uk).

Digital Object Identifier 10.1109/TED.2006.876039
A fine mesh is required to account for steep gradients of the order parameter in proximity to disclinations, which severely limits the time step particularly in the case of explicit methods. An implicit time-integration scheme is used here to alleviate this problem. Using the finite elements with an adaptive meshing scheme, the mesh density can be concentrated about disclinations, affording significant savings in terms of the number of degrees of freedom compared to the traditional finite-difference methods.

This paper details a finite-element implementation of the Qian and Sheng equations. An approach to obtain the steady state $Q$-tensor field is outlined and then generalized for the dynamic case, including the flow-induced reorientation of the LC. The flow of the LC is found by solving a weak form of the Navier-Stokes equation. Finally, the model is validated with previously published results; specifically, the pair annihilation of $\pm 1 / 2$ disclination lines.

\section{II. $Q$-Tensor Statics}

In this section, a formulation for the steady state $Q$-tensor field is derived from the governing equations. A convergence analysis of these equations is given in [10]. The Landau-de Gennes (LdG) free energy functional [11], [12] is defined as

$$
\begin{aligned}
\mathcal{F}(\mathbf{Q}) & :=\mathcal{F}_{D}(\partial \mathbf{Q})+\mathcal{F}_{B}(\mathbf{Q})-\mathcal{F}_{E}(\mathbf{Q})+\mathcal{F}_{S}(\mathbf{Q}) \\
& =\int_{\Omega}\left\{f_{D}(\partial \mathbf{Q})+f_{B}(\mathbf{Q})-f_{E}(\mathbf{Q})\right\}+\int_{\Gamma}\left\{f_{S}(\mathbf{Q})\right\}
\end{aligned}
$$

where $\Omega$ is an open bounded subset of $\mathbf{R}^{3}$ with boundary $\Gamma$, and $\mathbf{Q}$ is as those in [4] and [13]. The free-energy densities, $f_{B}(\mathbf{Q})$, and $f_{D}(\partial \mathbf{Q})$ are due to bulk and elastic contributions, respectively, and $f_{S}(\mathbf{Q})$ is the surface free-energy density. Reorientation of the LC is induced by an applied electric field, and, thus, a linear term $f_{E}(\mathbf{Q})$, which is the electrostatic energy density, is also included.

The bulk free-energy density determines the state of the LC, be it uniaxial or biaxial nematic or isotropic. It can be written as an expansion in the scalar invariants of the $Q$ tensor about the nematic-isotropic transition, truncated to include at most fourth-order terms:

$$
f_{B}(\mathbf{Q}):=\frac{1}{2} A \operatorname{tr}\left(\mathbf{Q}^{2}\right)+\frac{1}{3} B \operatorname{tr}\left(\mathbf{Q}^{3}\right)+\frac{1}{4} C \operatorname{tr}\left(\mathbf{Q}^{2}\right)^{2}
$$

where $A, B$, and $C$ are the material bulk constants [11]. The truncation used is sufficient to describe the $Q$-tensor field due to a disclination: a biaxial arrangement of uniaxial molecules 
lying in a ring about the disclination core [14]. More general densities include higher order terms and allow such states as the bulk biaxial phase [11].

The distortion energy density is quadratic in the gradients of the $Q$ tensor

$$
f_{D}(\partial \mathbf{Q}):=\frac{1}{2} L_{1} Q_{\alpha \beta, \gamma} Q_{\alpha \beta, \gamma}+\frac{1}{2} L_{2} Q_{\alpha \beta, \beta} Q_{\alpha \gamma, \gamma} .
$$

For simplicity of presentation, the splay-bend anisotropy and chiral terms [13] have been omitted from this description.

To describe weak surface anchoring, a simple penaltylike method can be used to enforce alignment

$$
f_{S}(\mathbf{Q}):=\frac{1}{2} W \operatorname{tr}\left(\left(\mathbf{Q}-\mathbf{Q}_{0}\right)^{2}\right)
$$

where $\mathbf{Q}_{0}$ is the prescribed $Q$ tensor on the boundary, and $W$ is the anchoring strength. A more advanced form is used in our model, with a different penalty associated with azimuthal and zenithal deformations, given by an expansion in the components of the $Q$ tensor.

The electrostatic energy is linear in the components of the $Q$ tensor and is written in terms of the electric field $\vec{E}$

$$
f_{E}(\mathbf{Q}):=\frac{1}{2} \varepsilon_{0}(\vec{E} \cdot \overline{\bar{\varepsilon}} \cdot \vec{E}) .
$$

In order to solve these equations, the symmetry and zero trace of the $Q$ tensor should be maintained. This can be achieved by several means. Lagrange multipliers can be used to enforce the condition, expanding the solution vector. This expansion can be avoided using a penalty method, but, still, all nine components of the $Q$ tensor would have to be found. If an explicit timestepping procedure is employed, these complications can be avoided by projecting the $Q$-tensor field onto a traceless and symmetric subspace at each step [7]. Most efficiently, $\mathbf{Q}$ can be written in terms of a five-dimensional subspace, defined by an orthonormal basis [15]

$$
\mathbf{Q}=q_{i} \mathbf{T}_{i}
$$

There are many possible choices for the basis tensors $\mathbf{T}_{i}$; we use one from [15]. This choice maintains the symmetry and the zero trace of the $Q$ tensor and enables us to calculate the steady state or dynamic solutions

$$
\begin{aligned}
\mathbf{T}_{1} & =\left(3 \hat{\mathbf{e}}_{z} \hat{\mathbf{e}}_{z}-\mathbf{I}\right) / \sqrt{6} \\
\mathbf{T}_{2} & =\left(\hat{\mathbf{e}}_{x} \hat{\mathbf{e}}_{x}-\hat{\mathbf{e}}_{y} \hat{\mathbf{e}}_{y}\right) / \sqrt{2} \\
\mathbf{T}_{3} & =\left(\hat{\mathbf{e}}_{x} \hat{\mathbf{e}}_{y}+\hat{\mathbf{e}}_{y} \hat{\mathbf{e}}_{x}\right) / \sqrt{2} \\
\mathbf{T}_{4} & =\left(\hat{\mathbf{e}}_{x} \hat{\mathbf{e}}_{z}+\hat{\mathbf{e}}_{z} \hat{\mathbf{e}}_{x}\right) / \sqrt{2} \\
\mathbf{T}_{5} & =\left(\hat{\mathbf{e}}_{y} \hat{\mathbf{e}}_{z}+\hat{\mathbf{e}}_{z} \hat{\mathbf{e}}_{y}\right) / \sqrt{2}
\end{aligned}
$$

where $\hat{\mathbf{e}}_{x}, \hat{\mathbf{e}}_{y}$, and $\hat{\mathbf{e}}_{z}$ are unit vectors along $x, y$, and $z . \mathbf{T}_{1}$ describes changes in the order parameter; $\mathbf{T}_{2}$ describes the changes in the biaxiality, and $\mathbf{T}_{3}, \mathbf{T}_{4}$, and $\mathbf{T}_{5}$ correspond to rotations [16]. Due to the normalization, the basis tensors satisfy the orthogonality condition: $\operatorname{tr}\left(\mathbf{T}_{i} \mathbf{T}_{j}\right)=\delta_{i j}$. From (1) and (3)

$$
\begin{aligned}
f_{B}=\frac{A}{2} q_{k} q_{k} & +\frac{C}{4}\left(q_{k} q_{k}\right)^{2}+\frac{B}{2 \sqrt{2}}\left[q_{2}\left(q_{4}^{2}-q_{5}^{2}\right)+2 q_{3} q_{4} q_{5}\right] \\
& +\frac{B}{6 \sqrt{6}} q_{1}\left[2 q_{1}^{2}+3\left(q_{4}^{2}+q_{5}^{2}-2 q_{2}^{2}-2 q_{3}^{2}\right)\right] .
\end{aligned}
$$

From (2) and (3), and considering, for brevity, a twodimensional problem oriented in the $x-z$ plane, the elastic energy can be reexpressed as

$$
\begin{aligned}
f_{D}= & \frac{L_{1}}{2} q_{k, \alpha} q_{k, \alpha}+\frac{L_{2}}{4}\left[q_{2, x}^{2}+q_{3, x}^{2}+q_{4, x}^{2}+q_{4, z}^{2}+q_{5, z}^{2}\right] \\
& +\frac{L_{2}}{12}\left[q_{1, x}^{2}+4 q_{1, z}^{2}+6 q_{2, x} q_{4, z}+6 q_{3, x} q_{5, z}\right] \\
& +\frac{L_{2}}{2 \sqrt{3}}\left[2 q_{1, z} q_{4, x}-q_{1, x}\left(q_{2, x}+q_{4, z}\right)\right]
\end{aligned}
$$

The final step in the procedure is to represent the $Q$-tensor components $q_{k}$ and the potential $u(\vec{E}=-\nabla u)$ as a linear combination of shape functions (interpolation functions, piecewise polynomials) with amplitude coefficients chosen to approximate $q_{k}$ and $u$. It is advantageous to use second-order shape functions in both cases. The asymptotic error is of fourth order as opposed to second order for the first-order elements, giving a better accuracy for a given number of nodes. In each element, the discretized form of $q_{k}$ and $u$ can be written

$$
q_{k}=\sum_{i=1}^{N} q_{k}^{i} \phi^{i}, \quad u=\sum_{i=1}^{N} u^{i} \phi^{i}
$$

where $N$ is the number of nodes in the element, $\phi^{i}$ is the shape function, and $q_{k}^{i}$ and $u^{i}$ are the amplitude coefficients to be found. Instead of directly minimizing the $\mathrm{LdG}$ free-energy functional, the computationally simpler task of seeking the $Q$ tensor field that renders the functional stationary is performed

$$
\frac{\partial \mathcal{F}}{\partial q_{k}^{i}}=0
$$

In order to determine the matrix form, a solution vector $\mathbf{q}=$ $\left\{q_{k}^{i}\right\}$ is introduced containing the values of all five $Q$-tensor components at each node. The nonlinear system (6) takes the form $[\mathbf{K}+\mathbf{B}(\mathbf{q})] \mathbf{q}=\mathbf{g}$, which can be solved using the Newton-Raphson method for $\Delta \mathbf{q}=\mathbf{q}^{m+1}-\mathbf{q}^{m}$, where $m$ denotes the iteration number, leading to

$$
\left[\mathbf{K}+\mathbf{J}\left(\mathbf{q}^{m}\right)\right] \Delta \mathbf{q}=\mathbf{g}-\left[\mathbf{K}+\mathbf{B}\left(\mathbf{q}^{m}\right)\right] \mathbf{q}^{m} .
$$

$\mathbf{K}$ contains terms independent of $\mathbf{q}$ arising from the distortion and surface energies, and the terms depending on $\mathbf{q}$ from the bulk energy are grouped in $\mathbf{B}$. The vector $\mathbf{g}$ comes from the electrostatic energy density. At each iteration, there is an overhead associated with the assembly of the Jacobian matrix $\mathbf{J}$, which is defined as

$$
J_{i j}=\frac{\partial\left(B_{i k} q_{k}\right)}{\partial q_{j}} .
$$


Iterations are performed until $\|\Delta \mathbf{q}\|<\zeta$ for a given tolerance $\zeta$. Convergence can be improved by using a line search algorithm; the right-hand side of (7) is scaled by a damping factor, which can be optimally chosen by a strategy such as the Armijo-Goldstein method [17], [18].

\section{Electric Potential}

To solve for the electric potential, an estimation of the permittivity of the LC medium is required in terms of $\mathbf{Q}$

$$
\varepsilon_{\alpha \beta}=\varepsilon_{\perp} \delta_{\alpha \beta}+\Delta \varepsilon\left(\frac{2}{3 S_{1}} Q_{\alpha \beta}+\frac{1}{3} \delta_{\alpha \beta}\right) .
$$

$\Delta \varepsilon$ is the dielectric anisotropy, and $\varepsilon_{\perp}$ is the permittivity in the direction perpendicular to the long axis of the molecule. In the uniaxial state, $\mathbf{Q}$ is defined as $S_{1}(3 \hat{n} \hat{n}-I) / 2$, where $\hat{n}$ is the director, and $S_{1}$ is the equilibrium value of the order parameter, which can be found from the stationary value of $f_{B}$, giving $S_{1}=\left(-B+\sqrt{B^{2}-24 A C}\right) /(6 C)$. The polarizability of the $\mathrm{LC}$ is neglected in this description, however, the inclusion complicates the model only slightly.

The electric field is calculated from the potential $u$, found by solving the weak form of Laplace's equation

$$
\int_{\Omega}\left\{u_{, \beta} \varepsilon_{\alpha \beta} \phi_{, \alpha}^{i}\right\}-\int_{\Gamma_{N}}\left\{\eta_{\alpha} u_{, \beta}\left(\varepsilon_{\alpha \beta}+\delta_{\alpha \beta}\right) \phi^{i}\right\}=0 .
$$

The additional boundary integral over $\Gamma_{N}$ is necessary to obtain the desired Neumann boundary condition: $\hat{\eta} \cdot \nabla u=0$, where $\hat{\eta}$ is normal to the boundary. The expression above can be written in matrix form as

$$
\mathbf{S u}=\mathbf{b} .
$$

\section{IV. $Q$-TENSOR DYNAMICS}

The dynamics of LC switching are not merely governed by electric and elastic forces; reorientation of the LC is influenced by its flow. To obtain the dynamic solution, the weak form is supplemented with a term that accounts for both effects

$$
\frac{\partial \mathcal{F}}{\partial Q_{\alpha \beta}^{i}}+\int_{\Omega}\left\{H_{\alpha \beta} \phi^{i}\right\}=0
$$

with $H_{\alpha \beta}=(1 / 2) \mu_{2} A_{\alpha \beta}+\mu_{1} N_{\alpha \beta}$ and $A_{\alpha \beta}=(1 / 2)\left(\partial_{\alpha} v_{\beta}+\right.$ $\left.\partial_{\beta} v_{\alpha}\right)$, where $v_{\alpha}$ is the velocity field, $\mu_{1}$ and $\mu_{2}$ are viscosity coefficients, and $N_{\alpha \beta}=d Q_{\alpha \beta} / d t+W_{\alpha \mu} Q_{\mu \beta}-Q_{\alpha \mu} W_{\mu \beta}$. The total time-derivative operator is defined as $d / d t=(\partial / \partial t+$ $\vec{v} \cdot \nabla)$ and $W_{\alpha \beta}=(1 / 2)\left(\partial_{\alpha} v_{\beta}-\partial_{\beta} v_{\alpha}\right)$. To obtain the weak form in terms of $q_{k}$, (9) can be written as

$$
\frac{\partial \mathcal{F}}{\partial q_{k}^{i}}+\int_{\Omega}\left\{\operatorname{tr}\left(\mathbf{T}_{k} \mathbf{H}\right) \phi^{i}\right\}=0 .
$$

Numerical integration in time is performed using the Crank-Nicholson method. Due to the nonlinearity of the governing equations, Newton iterations are performed within each time step. Using $n$ to denote the time step and $m$ for the iteration number, the matrix form can be written as

$$
\begin{aligned}
{\left[\frac{2 \mu_{1}}{\Delta t} \mathbf{M}+\right.} & \left.\overline{\mathbf{K}}+\mathbf{J}\left(\mathbf{q}_{n+1}^{m}\right)\right] \Delta \mathbf{q}_{n+1}=\mu_{1} \mathbf{M}\left[\frac{2}{\Delta t} \mathbf{q}_{n}+\dot{\mathbf{q}}_{n}\right] \\
& +\overline{\mathbf{g}}_{n+1}-\left[\frac{2 \mu_{1}}{\Delta t} \mathbf{M}+\overline{\mathbf{K}}+\mathbf{B}\left(\mathbf{q}_{n+1}^{m}\right)\right] \mathbf{q}_{n+1}^{m} .
\end{aligned}
$$

The right-hand side is written in terms of the solution at the last time step, $\dot{\mathbf{q}}=\partial \mathbf{q} / \partial t$, and this form provides damping of roundoff errors [19], [20]. The matrix $\mathbf{M}$ is $\int_{\Omega}\left\{\phi^{i} \phi^{j}\right\}$. $\mathbf{g}$ is made up of $\mathbf{g}$ and velocity terms from $\mathbf{A} ; \overline{\mathbf{K}}$ comprises $\mathbf{K}$ and velocity terms from $\mathbf{W}$ (which renders $\overline{\mathbf{K}}$ asymmetric). Here, $\mathbf{g}, \mathbf{K}$, and $\mathbf{B}$ are as used in Section II.

\section{FLOW FORMULATION}

In this section, a finite-element discretization of the velocity-pressure form of the incompressible Navier-Stokes equation is described. The momentum equation, which is written in terms of a generalized stress tensor $\sigma$, is

$$
\rho \frac{d v_{\alpha}}{d t}=\partial_{\beta}\left(-p \delta_{\beta \alpha}+\sigma_{\beta \alpha}^{d}+\sigma_{\beta \alpha}^{e}+\sigma_{\beta \alpha}^{v}\right) .
$$

The pressure field $p$ is such that it satisfies the continuity equation, acting as a Lagrange multiplier to enforce the incompressibility of the fluid

$$
\partial_{\mu} v_{\mu}=0 .
$$

There are several contributions to the overall stress tensor: $\sigma_{\alpha \beta}^{v}$ is the viscous stress; $\sigma_{\alpha \beta}^{e}$ is the electric field-induced stress; and $\sigma_{\alpha \beta}^{d}$ is the distortion stress

$$
\begin{array}{r}
\sigma_{\alpha \beta}^{v}=\beta_{1} Q_{\alpha \beta} Q_{\mu \nu} A_{\mu \nu}+\beta_{4} A_{\alpha \beta}+\beta_{5} Q_{\alpha \mu} A_{\mu \beta}+\beta_{6} Q_{\beta \mu} A_{\mu \alpha} \\
+\frac{1}{2} \mu_{2} N_{\alpha \beta}-\mu_{1} Q_{\alpha \mu} N_{\mu \beta}+\mu_{1} Q_{\beta \mu} N_{\mu \alpha} .
\end{array}
$$

The viscosity coefficients $\left\{\mu_{1}, \mu_{2}, \beta_{1}, \beta_{4}, \beta_{5}, \beta_{6}\right\}$ can be obtained from the Ericksen-Leslie coefficients $\left\{\alpha_{1}, \alpha_{2}, \ldots, \alpha_{6}\right\}$ using the mappings given in [13] and [4]. The expression for the electric field-induced stress tensor is simplified assuming that LC is incompressible

$$
\sigma_{\alpha \beta}^{e}=D_{\alpha} E_{\beta}-\frac{1}{2} D_{\mu} E_{\mu} \delta_{\alpha \beta}
$$

where $\vec{D}$ is the electric displacement. The distortion stress is given by

$$
\sigma_{\alpha \beta}^{d}=-\frac{\partial f_{D}}{\partial\left(Q_{\mu \nu, \alpha}\right)} Q_{\mu \nu, \beta} .
$$

The weak form is obtained taking a Galerkin approach, giving a set of three coupled equations for the $x, y$, and $z$ components of the velocity

$$
\int_{\Omega}\left\{\rho \phi^{i} \frac{d v_{\alpha}}{d t}\right\}=\int_{\Omega}\left\{\phi^{i} \partial_{\beta}\left(-p \delta_{\beta \alpha}+\sigma_{\beta \alpha}^{d}+\sigma_{\beta \alpha}^{e}+\sigma_{\beta \alpha}^{v}\right)\right\} .
$$


The shape functions here are the same second-order functions used to represent the $Q$-tensor components. Similarly, for the pressure

$$
\int_{\Omega}\left\{\psi^{i} \partial_{\mu} v_{\mu}\right\}=0
$$

A different set of shape functions $\psi$ of lower order are used for the pressure. If they are of the same order as those for the velocity, too many constraints are placed upon the velocity, leading to oscillatory solutions for the pressure [20]. An alternative to this mixed interpolation procedure is using a formulation stabilized by the inclusion of damping terms.

The momentum equation is integrated by parts, yielding

$$
\int_{\Omega}\left\{\rho \phi^{i} \frac{d v_{\alpha}}{d t}\right\}=-\int_{\Omega}\left\{\phi_{, \beta}^{i}\left(-p \delta_{\beta \alpha}+\sigma_{\beta \alpha}^{d}+\sigma_{\beta \alpha}^{e}+\sigma_{\beta \alpha}^{v}\right)\right\} .
$$

The surface terms that arise from the integration are set to zero, leading to the natural boundary condition

$$
\eta_{\beta}\left(-p \delta_{\beta \alpha}+\sigma_{\beta \alpha}^{d}+\sigma_{\beta \alpha}^{e}+\sigma_{\beta \alpha}^{v}\right)=0
$$

for the velocity components. For nonslip or periodic boundaries, this surface term can be neglected, but if any part of the boundary remains free, then, this boundary integral should be modified appropriately. No pressure boundary condition is implied in the above formulation; the pressure at the boundary merely satisfies the incompressibility condition as required.

The use of a numerical-integration scheme allows some simplification of these expressions. The weak form of the momentum equation can be rearranged as

$$
\int_{\Omega}\left\{\rho \phi^{i} \frac{d v_{\alpha}}{d t}-p \phi_{, \alpha}^{i}+\phi_{, \beta}^{i}\left(v_{\mu, \nu} G_{\beta \nu}^{\alpha \mu}+v_{\mu} U_{\beta}^{\alpha \mu}+V_{\beta}^{\alpha}\right)\right\}=0
$$

where $G_{\beta \nu}^{\alpha \mu}$ contains the material viscosity coefficients and the components of the $Q$ tensor $q_{k}$, and $U_{\beta}^{\alpha \mu}$ is made up of gradients in $q_{k} . V_{\beta}^{\alpha}$ contains the driving-stress components due to the reorientation of the LC, the distortion stress, and the fieldinduced stress.

For elasticity-driven dynamics of LCs, the low Reynoldsnumber approximation can be made, whereby the nonlinear advective term $(\vec{v} \cdot \nabla) \vec{v}$ arising from the total time derivative is neglected. An unsteadiness parameter can be defined as the ratio of the characteristic times of $Q$-tensor and velocity fields [7]. When studying disclination dynamics, the velocity field adapts quickly to a given $Q$-tensor field. A small unsteadiness parameter results, and the partial time derivative can be neglected from (11). Typically, these two approximations are valid in case of conventional cell dynamics (in the absence of disclinations). However, in extreme cases, with large electric fields and large container sizes, this unsteadiness parameter is no longer small. A time-stepping procedure, such as (10), may be used to calculate the velocity field with the pressure assumed steady within each time step so that the incompressibility condition holds for all time.
After discretizing the velocity and pressure using $v_{\alpha}=v_{\alpha}^{i} \phi^{i}$ and $p=p^{i} \psi^{i}$, the system of equations may be written in matrix form as

$$
\left(\begin{array}{cc}
\mathbf{D} & \mathbf{C} \\
\mathbf{C}^{T} & 0
\end{array}\right)\left(\begin{array}{l}
\mathbf{v} \\
\mathbf{p}
\end{array}\right)=\left(\begin{array}{l}
\mathbf{f}_{1} \\
\mathbf{f}_{2}
\end{array}\right)
$$

assuming that both the advective term and the partial time derivative can be dropped. The submatrices $\mathbf{D}$ and $\mathbf{C}$ arise from the terms in $\vec{v}$ and $p$, respectively. The vectors $\mathbf{f}_{1}$ and $\mathbf{f}_{2}$ arise from $V_{\beta}^{\alpha}$ and the Dirichlet boundary conditions applied to the velocity and pressure solutions and $\mathbf{v}=\left\{v_{\alpha}^{i}\right\}$. A scaled pressure vector $\mathbf{p}$ is introduced to improve the condition number of the system matrix. In the above formulation, the choice was made to integrate the pressure term by parts, but this operation is not essential. It has the advantage that the resultant system matrix is symmetric, but the real difference is seen on open boundaries. This procedure modifies the natural boundary condition so that it is well posed on open boundaries. The resulting weak form contains no derivatives of the pressure, introducing the possibility to represent the pressure by a function that is not $C^{0}$ continuous. Improved convergence is reported in [20] using these functions that allow the continuity equation to be satisfied on an element-by-element basis.

Two element types have been tested: First, the P2P1 TaylorHood [21] element with second-order functions for the velocity components and continuous linear functions for the pressure, and, second, the $\mathrm{P} 2(\mathrm{P} 1+\mathrm{P} 0)$ (as used to solve the Ericksen-Leslie equations in [22]) with second-order functions for the velocity components and a discontinuous linear approximation for the pressure. The implementation of the later is more complicated, and the solution vector is extended by the number of elements in the mesh. More complicated elements, using bubble functions, are common in the field of computational fluid dynamics, but these lead to a much expanded solution vector and a more complex implementation. The P2P1 element was found to give a solution vector $(\mathbf{v})$ differing by $\left\|\mathbf{v}-\mathbf{v}^{\prime}\right\|_{\infty} \approx 10^{-5}$ compared to the $\mathrm{P} 2(\mathrm{P} 1+\mathrm{P} 0)$ element $\left(\mathbf{v}^{\prime}\right)$. This difference is not substantial, therefore, the simpler P2P1 element is used.

\section{Algorithm Details}

The equations are put into dimensionless form by introducing a characteristic length and time scale. These parameters depend on the bulk-energy coefficients and the elastic constants. The characteristic length $\xi$, which is also referred to as the correlation length, is typically a few nanometers, and the characteristic time $\tau$ is typically tens of nanoseconds

$$
\xi=\sqrt{\frac{27 C L_{1}}{B^{2}}} \quad \tau=\mu_{1} \frac{\xi^{2}}{L_{1}}
$$

Detailed expressions of $\mathbf{K}, \mathbf{B}$, and $\mathbf{g}$ have been found using Maple, starting from

$$
\mathbf{K q}=\frac{\partial \mathcal{F}_{D}}{\partial q_{k}^{i}} \quad \mathbf{B q}=\frac{\partial \mathcal{F}_{B}}{\partial q_{k}^{i}} \quad \mathbf{g}=\frac{\partial \mathcal{F}_{E}}{\partial q_{k}^{i}}
$$


A similar procedure is used to obtain $G_{\beta \nu}^{\alpha \mu}, U_{\beta}^{\alpha \mu}$, and $V_{\beta}^{\alpha}$ for the calculation of the velocity field.

To summarize the discussions made in the previous sections, second-order polynomials are chosen as the shape functions for $q_{k}, u$, and $\vec{v}$, and linear (continuous) functions for the pressure. Integration is performed numerically using Gauss quadrature, opening up the possibility to use isoparametric elements, which are better able to conform to the curved boundaries. For the $Q$-tensor problem in 2D, 16 Gauss points are chosen to avoid integration loss, giving eighth-order accuracy.

The system of (8), (10), and (12) is solved using Algorithm 1. A time-stepping procedure for the $Q$ tensor forms the basis for the algorithm, and within each time step, Newton iterations are performed to deal with the nonlinear terms, using $\zeta \approx 10^{-6}$. In each time step, the flow and the potential solutions are assumed steady.The most time consuming part of the procedure is the dynamic calculation of the $Q$ tensor. An incomplete LU factorization is used for preconditioning, and the resultant system of equations is solved using a generalized minimum residual method [23]. The execution time for the iterative method is almost independent of the matrix bandwidth; however, this is not the case for the calculation of the preconditioner. Savings are achieved by applying a bandwidth reduction technique such as a symmetric reverse Cuthill-McKee permutation. Furthermore, the preconditioner can be kept constant during the Newton iterations for the $Q$ tensor, with no significant loss of precision.

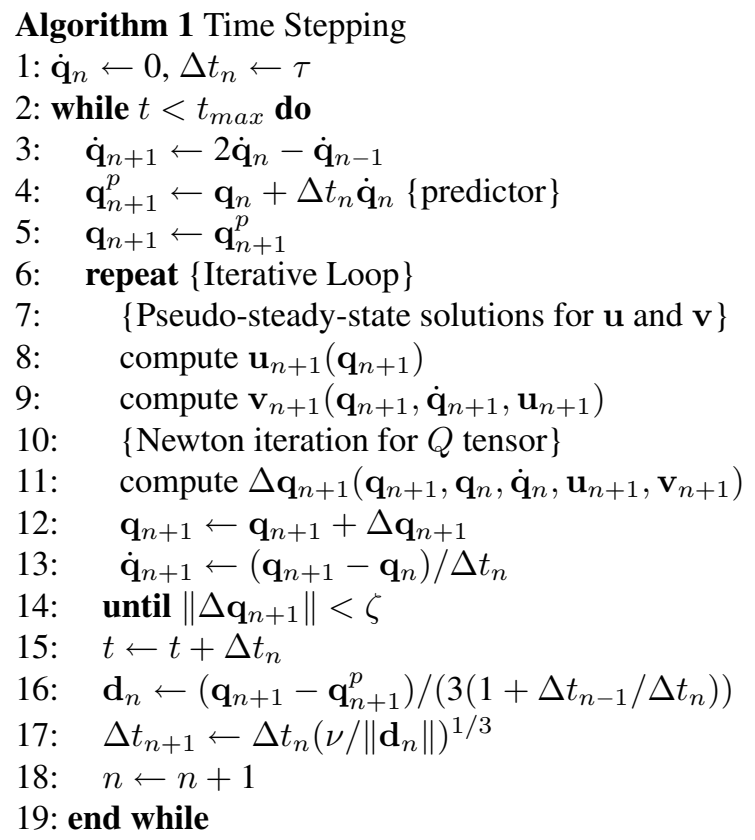

The $Q$ tensor is a function of the velocity and potential at step $n+1$. Strictly speaking, two loops are required: one to ensure consistency and the other to deal with the nonlinear terms. In practice, since the calculation of the $Q$ tensor is the bottleneck, it is preferable to update the pseudosteady-state values of the velocity and potential fields during the Newton iterations for the $Q$-tensor field.

Time integration is performed by an implicit method, therefore, a large time step may be used. However, due to the nonlinearity of the equations, some limit is imposed. When a voltage is applied, the $Q$ tensor changes rapidly and a small time step should be used to capture the transient behavior. After some time, as steady state is approached, a much larger time step may be used. A variable time-stepping procedure [19], [20] provides an effective means to calculate the dynamic behavior. In Algorithm 1, the time-integration error is maintained below a threshold $\nu$ by changes to the time step $\Delta t_{n}$. We have found that $\nu \approx 10^{-4}$ is a good compromise between computation time and accuracy.

Changes to the order parameter not only occur on small time scales, but length scales too. Such changes occur in proximity to disclinations, where it is important to use a fine mesh in order to calculate accurately the induced flow. Adaptive meshing is used solve this problem efficiently, particularly when calculating the motion of disclinations over large distances.

\section{VALIDATION}

In this section, the results of validation tests are compared to some previously published results [7], [14], [24] performed using a single elastic constant approximation $\left(L_{2}=0\right)$ and the material parameters of 4-methoxybenzylidene-4-butylanaline (MBBA) [11], [25]

$$
\begin{aligned}
a & =8.67 \times 10^{4} \mathrm{~N} / \mathrm{m}^{2} \cdot \mathrm{K}, \quad\left(T-T^{*}\right)=-4 \\
A & =a\left(T-T^{*}\right) \\
B & =-2.12 \times 10^{6} \mathrm{~N} / \mathrm{m}^{2} \\
C & =1.74 \times 10^{6} \mathrm{~N} / \mathrm{m}^{2} \\
\gamma_{1} & =0.0763 \mathrm{~Pa} \cdot \mathrm{s}, \quad \gamma_{2}=-0.0787 \mathrm{~Pa} \cdot \mathrm{s} \\
\alpha_{1} & =0.0065 \mathrm{~Pa} \cdot \mathrm{s}, \quad \alpha_{4}=0.0832 \mathrm{~Pa} \cdot \mathrm{s} \\
\alpha_{5} & =0.0463 \mathrm{~Pa} \cdot \mathrm{s}, \quad \alpha_{6}=-0.0344 \mathrm{~Pa} \cdot \mathrm{s} \\
k & =6 \mathrm{pN}, \quad \rho=10^{3} \mathrm{~kg} / \mathrm{m}^{3} .
\end{aligned}
$$

The $Q$ tensor and flow solver are first tested individually and then in combination.

\section{A. Q-Tensor Formulation Validation}

The $Q$-tensor formulation was tested for two simple cases: a planar and a twisted cell. Both static and dynamic director profiles were found to agree well with the previously verified constant-order-parameter model, based on a vectorial representation of the director [26].

To verify that the model can simulate variations of the order parameter appropriately, it is insightful to study disclinations. A starting director configuration with a $+1 / 2$ disclination at the center of the modeling window was chosen, and the iterations were performed until the $Q$ tensor reached a steady state. Such disclinations have been extensively studied (see, e.g., [14], [24]). Fig. 1 shows the variation in the two order parameters as a function of the distance $(r)$ from the disclination core.

At the disclination core $(r=0)$, the two order parameters are equal, and the LC is in the uniaxial nematic state. Encircling the disclination core is a ring of maximal biaxiality at approximately $r \approx 1 \mathrm{~nm}$. For large values of $r, \mathrm{LC}$ is again uniaxial. This behavior is an agreement with those in [14] and [24]. 


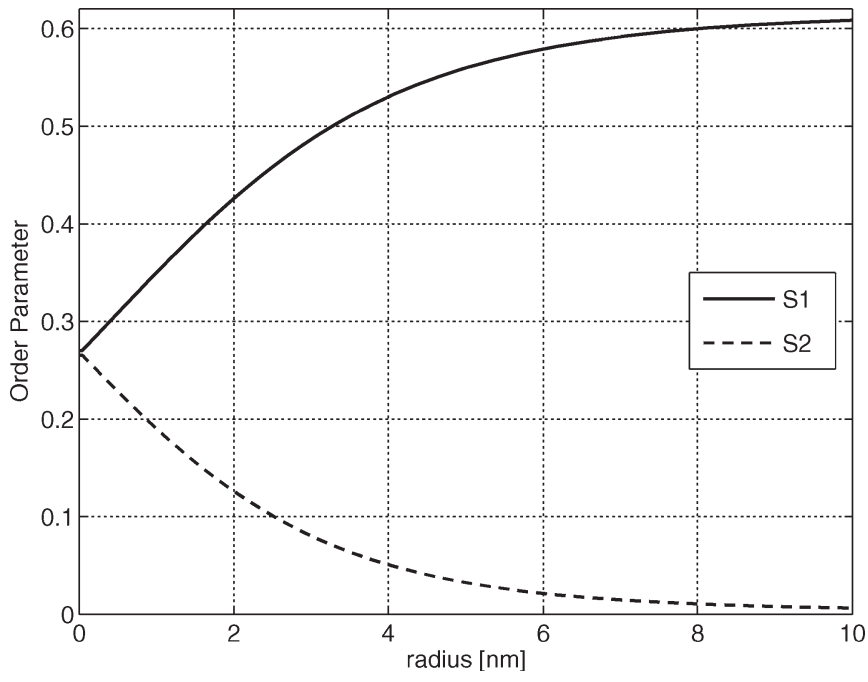

Fig. 1. Order parameters $S_{1}$ and $S_{2}$ as a function of radius from the core of a $+1 / 2$ disclination.

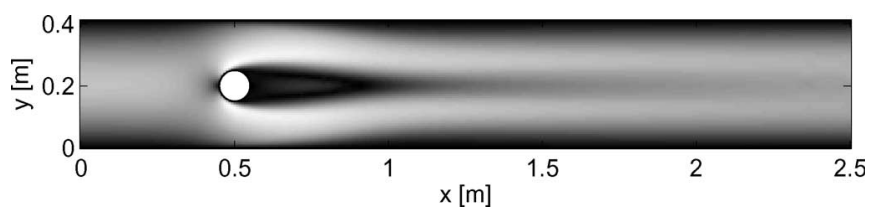

Fig. 2. Magnitude of the flow past a cylinder with a Reynolds number of 100 .

\section{B. Flow-Formulation Validation}

The flow around a cylinder [27] and the driven cavity problem [28] have been used to test the flow formulation. Such tests are normally performed using high Reynolds numbers where the advective term is important, or when the flow can no longer be assumed steady. Complications arise due to nonlinearities that, in general, can be neglected in the case of LC flow. For the study of flow around a cylinder, the dimensions are set according to [27], with a container size of $2.5 \mathrm{~m} \times$ $0.41 \mathrm{~m}$. At the point $(0.5,0.2) \mathrm{m}$, a cylinder of radius $0.1 \mathrm{~m}$ is centered. An isotropic liquid is considered in this benchmark test, and, so, all viscosity coefficients are set to zero except for $\beta_{4}=4 \cdot 10^{-4} \mathrm{~Pa} \cdot \mathrm{s}$. Nonslip conditions are applied to all boundaries with the exception of the inflow and outflow. At the inflow boundary, the incoming velocity is assumed to take a quadratic form $v_{x}=0.3 \cdot 4 y(0.41-y) / 0.41^{2}$, and, at the outflow boundary, the velocity is left free, and the pressure is set to zero. A mass density of $\rho=1.0 \mathrm{~kg} / \mathrm{m}^{3}$ is assumed. Convergence was achieved with Reynolds numbers in the region of 100, and the magnitude of the resulting flow is shown in Fig. 2. For higher Reynolds numbers, the velocity field can no longer be assumed steady.

\section{Validation of the Combined Formulation}

To test the combined formulation, we investigate the annihilation of $+1 / 2$ and $-1 / 2$ disclination lines. In [7], this process is studied for MBBA using a finite-difference discretization of the Qian and Sheng equations.

Disclinations are positioned along the $x$-axis, separated by a distance of $100 \mathrm{~nm}$. Much larger separations can be modeled by

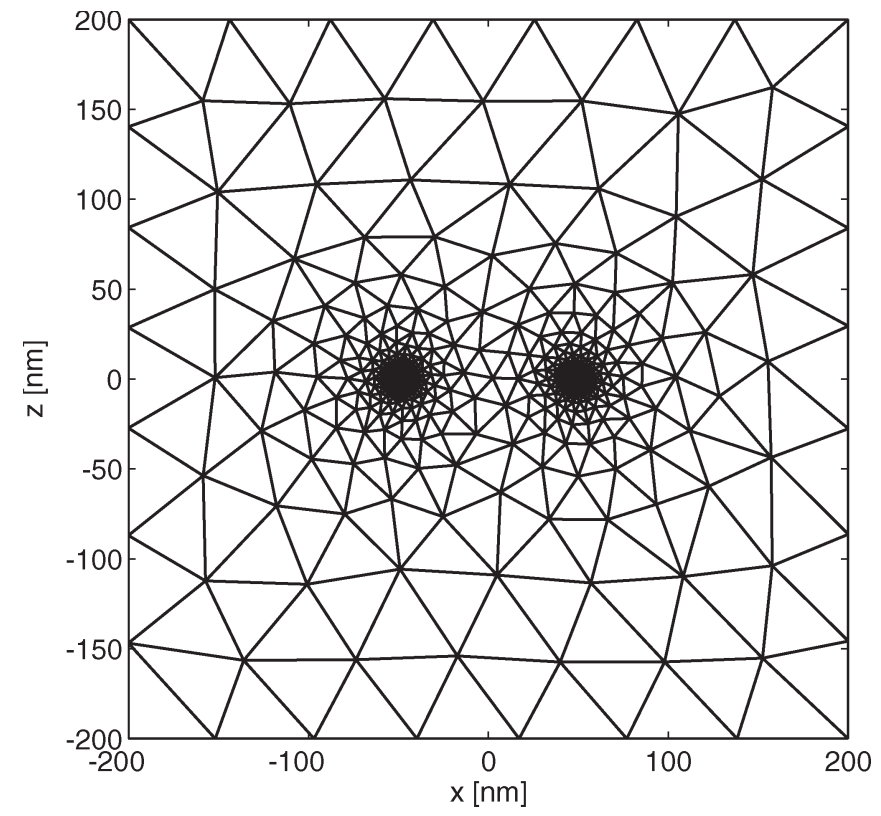

Fig. 3. Mesh for disclination-pair-annihilation problem. Ratio between the longest and shortest triangle edge is approximately 100:1.

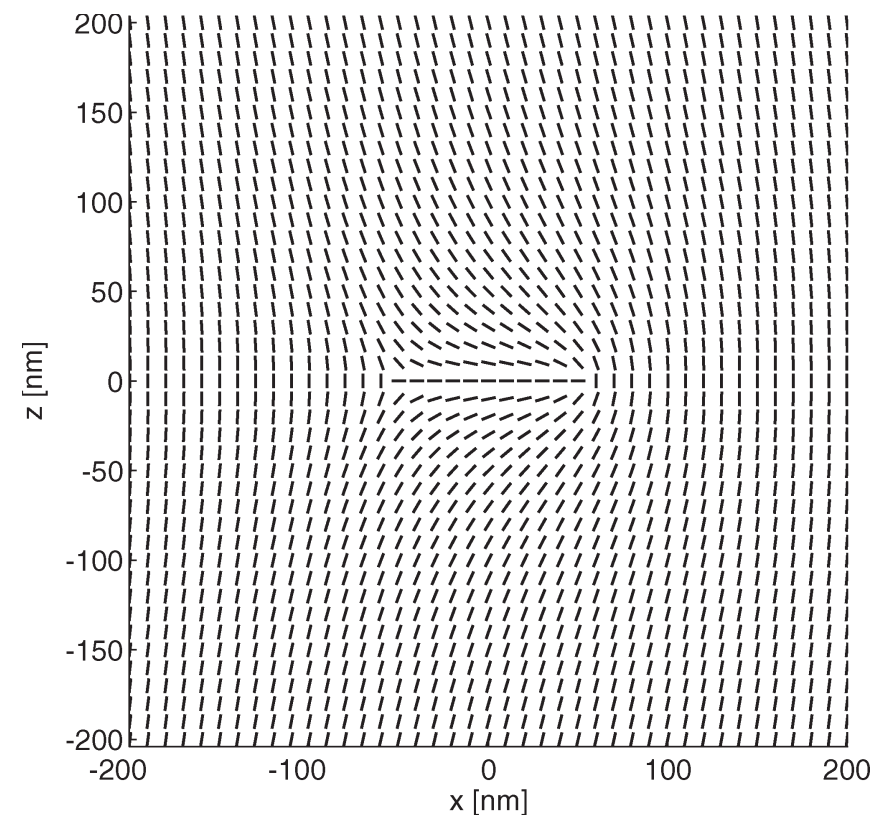

Fig. 4. Representation of the director profile for configuration (a) given by the eigenvector associated with the largest eigenvalue of $\mathbf{Q}$.

refining the mesh only in the vicinity of disclinations, as shown in Fig. 3. A large time step can be used when the disclinations are widely separated and slowly moving. However, when they are close and moving quickly, a small time step is necessary to follow the variations in the order parameter and flow.

There are two possible starting configurations depending on the placement of the two disclinations:

a) as shown in Fig. 4;

b) in which the initial director field is rotated by $90^{\circ}$.

The same boundary conditions are applied to all sides of the modeling window. For the $Q$ tensor, Neumann boundary conditions are applied - the natural boundary condition when 


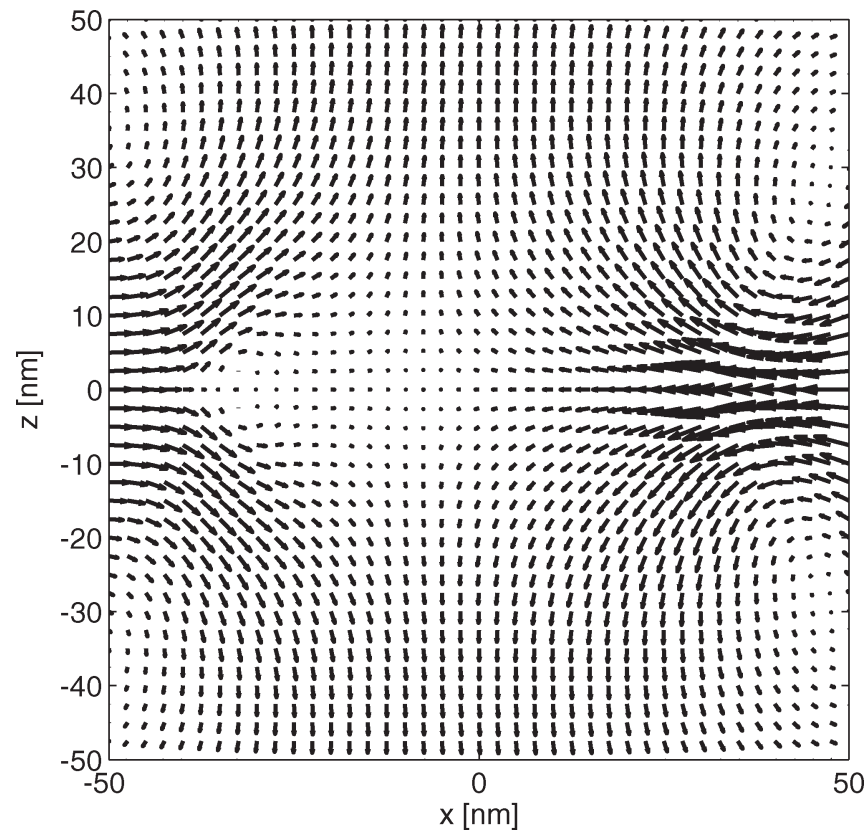

Fig. 5. Velocity field for a disclination separation of $75 \mathrm{~nm}$, assuming an isotropic fluid.

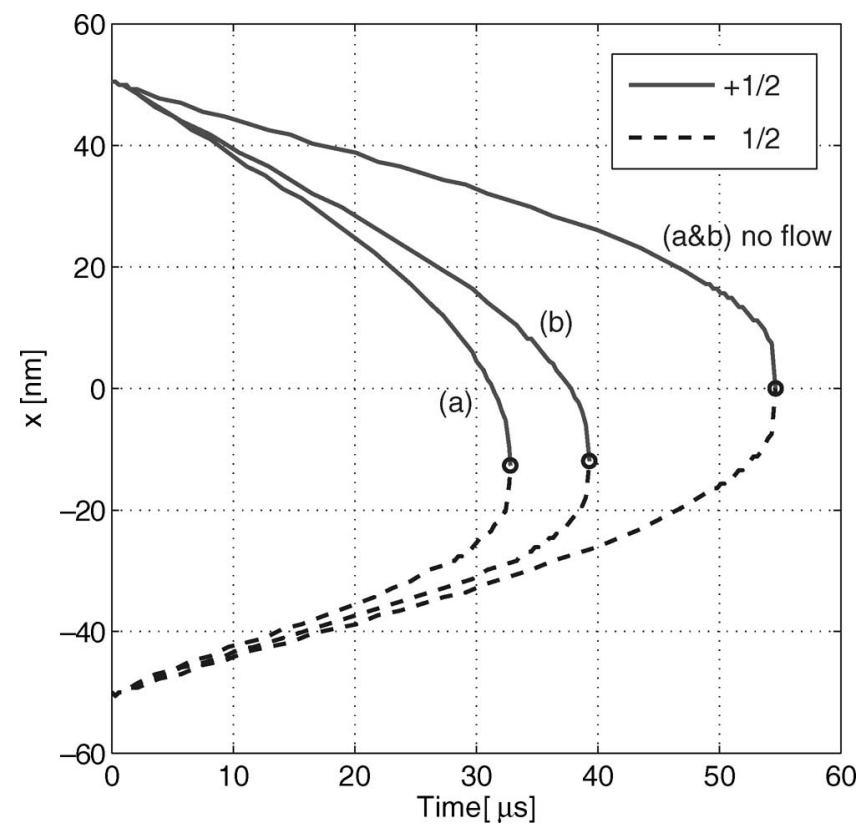

Fig. 6. Disclination position versus time for configurations (a) and (b) with and without flow.

using a single elastic constant formulation. For the velocity components, the nonslip condition is assumed to match the state in the bulk of the LC. No boundary condition is required for the pressure. Starting with $\mathbf{v}=0$, the steady-state velocity fields arising from the individual components of the driving-stress tensor were calculated and found to agree well with those in [7]. Fig. 5 shows the velocity field for the complete drivingstress tensor, for comparison with [7, Fig. 4(a)]. This field is highly sensitive to the disclination separation.

Fig. 6 shows the disclination position versus time for the two configurations with and without flow. This can be compared with [7, Fig. 2]. The velocity field serves to speed up preferentially the $+1 / 2$ disclination, an effect that has recently been observed experimentally [29]. It can be noted that for both configurations (a) and (b), the disclination path coincides in the absence of flow.

\section{CONCLUSION}

A finite-element model of the Qian and Sheng formulation, a generalization of the Ericksen-Leslie theory, allowing for changes in the order of the LC, has been presented and validated. The ability to adapt the mesh facilitates the modeling of realistically sized devices while still resolving small scale features and disclinations. This provides significant savings in terms of the number of degrees of freedom and computational cost in comparison to the traditional finite-difference methods. Time integration is performed using an implicit method, thus, additional savings are available through the use of a variable time step in accord with the rate of change of the LC orientation.

For the problem of disclination pair annihilation, this model agrees well with the previously published results, and the demonstrated increase in the $+1 / 2$ disclination velocity shows the importance of the LC flow when studying dynamic variations in the $Q$-tensor field.

\section{ACKNOWLEDGMENT}

The authors would like to thank Dr. D. Svenek for the helpful discussions.

\section{REFERENCES}

[1] D. W. Berreman, "Liquid crystal twist cell dynamics and backflow," J. Appl. Phys., vol. 46, no. 9, pp. 3746-3751, Sep. 1975.

[2] J. L. Ericksen, "Conservation laws for liquid crystals," J. Rheol., vol. 5, no. 1, pp. 23-34, Mar. 1961

[3] F. M. Leslie, "Some constitutive equations for liquid crystals," Arch. Ration. Mech. Anal., vol. 28, no. 4, pp. 265-283, Jan. 1968.

[4] T. Qian and P. Sheng, "Generalized hydrodynamic equations for nematic liquid crystals," Phys. Rev. E, Stat. Phys. Plasmas Fluids Relat. Interdiscip. Top., vol. 58, no. 6, pp. 7475-7485, Dec. 1998.

[5] A. N. Beris and B. J. Edwards, Thermodynamics of Flowing Systems. Oxford, U.K.: Oxford Univ. Press, 1994.

[6] G. Tóth, C. Denniston, and J. M. Yeomans, "Hydrodynamics of topological defects in nematic liquid crystals," Phys. Rev. Lett., vol. 88, no. 10, p. 105504, Mar. 2002.

[7] D. Svenšek and S. Žumer, "Hydrodynamics of pair-annihilating disclination lines in nematic liquid crystals," Phys. Rev. E, Stat. Phys. Plasmas Fluids Relat. Interdiscip. Top., vol. 66, no. 2, pt. 1, p. 021712, Aug. 2002.

[8] C. M. Care, I. Halliday, K. Good, and S. V. Lishchuk, "A generalised lattice Boltzmann algorithm for the flow of nematic liquid crystal with variable order parameter," Phys. Rev. E, Stat. Phys. Plasmas Fluids Relat. Interdiscip. Top., vol. 67, no. 6, p. 061703, Jun. 2003.

[9] J. I. Fukuda, "Effect of hydrodynamic flow on kinetics of nematicisotropic transition in liquid crystals," Eur. Phys. J., B, vol. 1, no. 2, pp. $173-177,1998$.

[10] T. A. Davis and E. C. Gartland, Jr., "Finite element analysis of the Landaude Gennes minimization problem for liquid crystals," SIAM J. Numer. Anal., vol. 35, no. 1, pp. 336-362, Feb. 1998.

[11] P. G. de Gennes and J. Prost, The Physics of Liquid Crystals, 2nd ed. Oxford, U.K.: Clarendon, 1993.

[12] L. D. Landau, E. M. Lifshitz, and L. P. Pitaevskii, Statistical Physics, 3rd ed. ser. Course of theoretical physics, vol. 5. Oxford, U.K.: Pergamon, 1980.

[13] H. Mori, E. C. Gartland, J. R. Kelly, and P. J. Bos, "Multidimensional director modeling using the $\mathrm{Q}$ tensor representation in a liquid crystal cell and its application to the $\pi$ cell with patterned electrodes," Jpn. J. Appl. Phys., vol. 38, no. 1A, pp. 135-146, Jan. 1999. 
[14] N. Schopohl and T. J. Sluckin, "Defect core structure in nematic liquid crystals," Phys. Rev. Lett., vol. 59, no. 22, pp. 2582-2584, Nov. 1987.

[15] A. Sonnet, A. Kilian, and S. Hess, "Alignment tensor versus director: Description of defects in nematic liquid crystals," Phys. Rev. E, Stat. Phys. Plasmas Fluids Relat. Interdiscip. Top., vol. 52, no. 1, pp. 718-722, Jul. 1995.

[16] D. Svenšek and S. Žumer, "Instability modes of high-strength disclinations in nematics," Phys. Rev. E, Stat. Phys. Plasmas Fluids Relat. Interdiscip. Top., vol. 70, no. 6, pt. 1, p. 061707, 2004.

[17] A. Goldstein, "On steepest descent," SIAM J. Control Optim., vol. 3, no. 1, pp. $147-151,1965$.

[18] L. Armijo, "Minimization of functions having Lipschitz continuous first partial derivatives," Pac. J. Math., vol. 16, no. 1, pp. 1-3, 1966.

[19] P. M. Gresho, R. L. Sani, and M. S. Engelman, Incompressible Flow and the Finite Element Method. Chichester, U.K.: Wiley, 2000.

[20] _ Incompressible Flow and the Finite Element Method. Chichester, U.K.: Wiley, 2000.

[21] P. Hood and C. Taylor, Navier-Stokes Equations Using Mixed Interpolation. Mexico City, Mexico: UAM Press, 1974.

[22] S. J. Tavener, T. Mullin, G. I. Blake, and K. A. Cliffe, "Numerical bifurcation study of electrohydrodynamic convection in nematic liquid crystals," Phys. Rev. E, Stat. Phys. Plasmas Fluids Relat. Interdiscip. Top., vol. 63, no. 1, pt. 1, p. 011708 , Jan. 2001.

[23] G. H. Golub and C. F. Van Loan, Matrix Computations, 3rd ed. ser. Johns Hopkins studies in the mathematical sciences. Baltimore, MD: The Johns Hopkins Univ. Press, 1996.

[24] N. J. Mottram and S. J. Hogan, "Disclination core structure and induced phase changes in nematic liquid crystals," Philos. Trans. Roy. Soc. London A, Math. Phys. Sci., vol. 355, no. 1731, pp. 2045-2064, Oct. 1997.

[25] H. J. Coles, "Laser and electric field induced birefringence studies on the cyanobiphenyl homologues," Mol. Cryst. Liq. Cryst., vol. 49, pp. $67-74,1978$.

[26] F. A. Fernández, S. E. Day, P. Trwoga, H. Deng, and R. James, "Threedimensional dynamic modelling of liquid crystal display cells using finite elements," Mol. Cryst. Liq. Cryst., vol. 375, pp. 291-299, 2002.

[27] M. Schäfer and S. Turek, "Benchmark computations of laminar flow around a cylinder," in Flow Simulation With High-Performance Computers II, Notes in Fluid Mechanics, vol. 52, E. H. Hirschel, Ed. Braunschweig, Germany: Vieweg, 1996, pp. 547-566.

[28] E. Erturk, T. C. Corke, and C. Gokcol, "Numerical solutions of 2-D steady incompressible driven cavity flow at high Reynolds numbers," Int. J. Numer. Methods Fluids, vol. 48, no. 7, pp. 747-774, 2005.

[29] C. Blanc, D. Svenšek, S. Žumer, and M. Nobili, "Dynamics of nematic liquid crystal disclinations: The role of the backflow," in Phys. Rev. Lett., Aug. 2005, vol. 95, p. 097802.

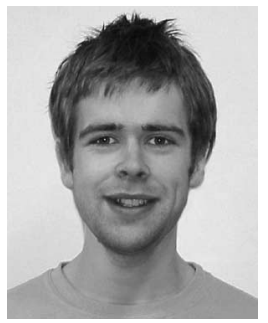

Richard James received the M.Eng. degree in electronic and electrical engineering from the University College London, London, U.K., in 2001. In 2003, he started working toward the Ph.D. degree at the same university.

He spent 18 months working as a Research Fellow in the Liquid Crystal Modelling Group, University College London. His research interests include modeling of LC devices for displays and communications.

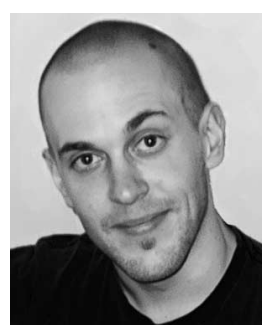

Eero Willman received the M.Eng. degree in electronic and electrical engineering from the Department of Electronic and Electrical Engineering, University College London, London, U.K., in 2003. $\mathrm{He}$ is currently working toward the Ph.D. degree at the same university.

His current research interests are computer modeling and development of modeling tools for the simulation of nematic LC devices.

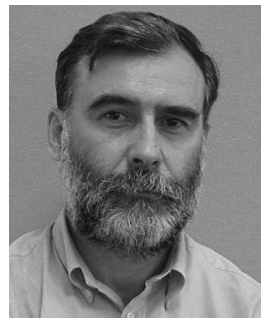

F. Aníbal Fernández (M'88) received the B.Sc. degree in applied mathematics from Universidad de Chile, Santiago, Chile, in 1969 and the Ph.D. degree in electrical engineering from the University College London, London, U.K., in 1981.

Until 1985, he was on the Academic Staff of the Department of Electrical Engineering, Universidad de Chile. In 1986, he joined the staff of the Department of Electronic and Electrical Engineering, University College London, London, U.K. where he is currently a Senior Lecturer. His research interests include microwave and optical aspects of electromagnetic theory, especially the use of numerical methods in those fields. In the last ten years, he has concentrated mainly on the modeling of LC devices.

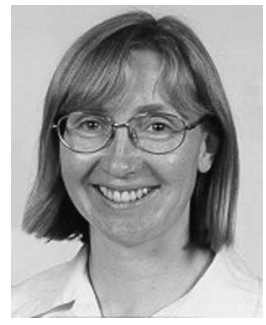

Sally E. Day (M'99) received the degree in physics from St. Hilda's College, Oxford University, Oxford, U.K., in 1983 and the D.Phil. degree from Oxford University, in 1988.

She spent five years with Thorn EMI, Central Research Laboratories (CRL), and Royal Signals and Radar Establishment (RSRE), Malvern, studying various aspects of the optical properties of LCs, including the nonlinear optical properties. In 1992, she received a Royal Society University Research Fellowship held in the Electronic and Electrical Engineering Department at University College London, London, U.K., where she is currently a Senior Lecturer. Her research interests include the application of LCs in display and nondisplay devices. 